\title{
Chiral molecule formation in interstellar ice analogs: alpha-aminoethanol $\mathrm{NH}_{2} \mathrm{CH}\left(\mathrm{CH}_{3}\right) \mathrm{OH}$
}

\author{
F. Duvernay, V. Dufauret, G. Danger, P. Theulé, F. Borget, and T. Chiavassa
}

\begin{abstract}
Université de Provence, Laboratoire de Physique des Interactions Ioniques et Moléculaires Centre de St-Jérôme, Avenue Escadrille Normandie-Niemen, 13397 Marseille, France e-mail: fabrice.duvernay@univ-provence.fr
\end{abstract}

Received 6 July 2010 / Accepted 22 August 2010

\begin{abstract}
Aims. Aminoalcohol molecules such as alpha-aminoethanol $\mathrm{NH}_{2} \mathrm{CH}\left(\mathrm{CH}_{3}\right) \mathrm{OH}$ may be aminoacid precursors. We attempt to characterize and detect this kind of molecules which is important to establish a possible link between interstellar molecules and life as we know it on Earth.

Methods. We use Fourier transform infrared (FTIR) spectroscopy and mass spectrometry to study the formation of alphaaminoethanol $\mathrm{NH}_{2} \mathrm{CH}\left(\mathrm{CH}_{3}\right) \mathrm{OH}$ in $\mathrm{H}_{2} \mathrm{O}: \mathrm{NH}_{3}: \mathrm{CH}_{3} \mathrm{CHO}$ ice mixtures. Isotopic substitution with ${ }^{15} \mathrm{NH}_{3}$ and ab-initio calculation are used to confirm the identification of alpha-aminoethanol.

Results. After investigating the thermal reaction of solid $\mathrm{NH}_{3}$ and acetaldehyde $\mathrm{CH}_{3} \mathrm{CHO}$ at low temperature, we find that this reaction leads to the formation of a chiral molecule, the alpha aminoethanol $\mathrm{NH}_{2} \mathrm{CH}\left(\mathrm{CH}_{3}\right) \mathrm{OH}$. For the first time, we report the infrared and mass spectra of this molecule. We also report on its photochemical behavior under VUV irradiation. We find that the main photoproduct is acetamide $\left(\mathrm{NH}_{2} \mathrm{COCH}_{3}\right)$. Data provided in this work indicates that alpha-aminoethanol is formed in one hour at $120 \mathrm{~K}$ and suggests that its formation in warm interstellar environments such as protostellar envelopes or cometary environments is likely.
\end{abstract}

Key words. astrochemistry - methods: laboratory - ISM: molecules

\section{Introduction}

In molecular clouds, the chemical composition of the interstellar material evolves as clouds collapse to form protostars, and eventually main-sequence stars with planets and comets. As infrared absorption studies of protostellar environments have shown, many molecules are frozen on the icy mantle of dust grains Whittet et al. (1996). Their heating by the protostar infrared photons, cosmic rays, and ultraviolet radiation changes the ice composition and triggers chemical reactions. Newly formed molecules can eventually be released into the gas phase or be incorporated into comets and planets van Dishoeck et al. (1998). Changes in the ice molecular composition during the star formation process can be monitored by studying the infrared absorption bands of molecules and they provide data to constrain chemistry models. Among all chemical reactions occurring in the interstellar medium, in this paper, we decided to focus more particularly on thermal reactions. To observe these reactions, they have to be thermodynamically favored and possess a low activation barrier in order to occur on a reasonable timescale. Several thermal reactions that are expected to occur in interstellar grains have already been studied and can be classified into two types. The first type contains acid-base reactions. Main examples of which are the reactivity between $\mathrm{HNCO}$ and $\mathrm{NH}_{3}$, which leads to $\mathrm{NH}_{4}{ }^{+} \mathrm{OCN}^{-}$Raunier et al. $(2003,2004)$, or the reaction between $\mathrm{HCOOH}$ and $\mathrm{NH}_{3}$ which forms $\mathrm{HCOO}^{-} \mathrm{NH}_{4}{ }^{+}$Shutte et al. (1999). These reactions have a very low activation barrier and are expected to occur at temperatures lower than $50 \mathrm{~K}$ in interstellar ices. The second type of thermal reaction is addition reactions involving aminolysis or hydrolysis reactions. For example, it has been shown that amine $\mathrm{RNH}_{2}$ and $\mathrm{CO}_{2}$ can react in a water ice environment to produce carbamates in relevant astrophysical conditions and timescales (Bossa et al. 2008, 2009a). The simplest aminoalcohol, aminomethanol $\mathrm{NH}_{2} \mathrm{CH}_{2} \mathrm{OH}$, has been recently thermally produced in interstellar ice analogs at temperatures lower than $100 \mathrm{~K}$ from a reaction between $\mathrm{NH}_{3}$ and $\mathrm{H}_{2} \mathrm{CO}$ (Bossa et al. 2009b).

In this paper, we experimentally study the reaction between ammonia $\mathrm{NH}_{3}$ and acetaldehyde $\mathrm{CH}_{3} \mathrm{CHO}$ in a water ice environment. Gas phase acetaldehyde was detected in the Sagittarius B2 molecular cloud by observing rotational transitions and is suspected to be present into ice mantles. We demonstrate that it reacts with ammonia to produce a chiral molecule: the alphaaminoethanol $\mathrm{NH}_{2} \mathrm{CH}\left(\mathrm{CH}_{3}\right) \mathrm{OH}$. The formation of this molecule within ices is very interesting because, except in meteorites (Cronin \& Pizzarello 1997, 1999), no chiral molecule has been detected in either the interstellar medium, comets, or planetary environments. Thus, the detection of a chiral molecule and a possible enantiomeric excess provide evidence of a link between interstellar molecules and life as we know it on Earth. Among the chiral molecules produced by the living world, aminoacids play an important role and alpha-aminoethanol $\mathrm{NH}_{2} \mathrm{CH}\left(\mathrm{CH}_{3}\right) \mathrm{OH}$ can be considered as a possible aminoacid precursor (Charnley et al. 2001; Courmier et al. 2005).

In this work, we use Fourier transform infrared (FTIR) spectroscopy and mass spectrometry to study the formation of alpha-aminoethanol $\mathrm{NH}_{2} \mathrm{CH}\left(\mathrm{CH}_{3}\right) \mathrm{OH}$ in $\mathrm{H}_{2} \mathrm{O}: \mathrm{NH}_{3}: \mathrm{CH}_{3} \mathrm{CHO}$ ice mixtures. Isotopic substitution with ${ }^{15} \mathrm{NH}_{3}$ and ab-initio calculation are used to confirm the identification of alphaaminoethanol. The vacuum ultraviolet (VUV) photolysis of alpha-aminoethanol gives acetamide $\mathrm{CH}_{3} \mathrm{CONH}_{2}$ as a photoproduct, which provides additional proof of alpha-aminoethanol 
identification. The possible formation of alpha-aminoethanol $\mathrm{NH}_{2} \mathrm{CH}\left(\mathrm{CH}_{3}\right) \mathrm{OH}$ in different interstellar environments is then discussed. The kinetic, thermodynamic, and infrared data provided in this work suggest its formation in warm interstellar environments such as protostellar envelopes or in cometary environments.

\section{Experimental and theoretical}

Ammonia is commercially available as a $99.9995 \%$ pure gas from Air Liquide. Acetaldehyde is bought under its liquid form from Aldrich and is gently heated up to be evaporated. The $\mathrm{H}_{2} \mathrm{O}: \mathrm{NH}_{3}: \mathrm{CH}_{3} \mathrm{CHO}$ gas mixtures are prepared at room temperature into two primary vacuum pumped mixing lines, the first one for the $\mathrm{H}_{2} \mathrm{O}: \mathrm{NH}_{3}$ mixture and the second one for $\mathrm{CH}_{3} \mathrm{CHO}$, to prevent any gas phase reaction occuring. They are then codeposited with a chosen ratio onto a gold-plated metal surface cooled to $10 \mathrm{~K}$ by a Model $21 \mathrm{CTI}$ cold head within a high vacuum chamber ( $\mathrm{ca} 10^{-8} \mathrm{mbar}$ ). The IR spectra are recorded between 4000 and $650 \mathrm{~cm}^{-1}$ in the transmission mode using a FTIR spectrometer. A typical spectrum has a $1 \mathrm{~cm}^{-1}$ resolution and is averaged over one hundred interferograms. The sample is warmed using a heating resistance and the temperature is controlled using a Lakeshore Model 331 temperature controller. The mass spectra are recorded using a MKS quadrupole mass spectrometer as the products are being desorbed. The ionization source is an $70 \mathrm{eV}$ impact electronic source and the mass spectra are recorded between 1 and 80 amu. The VUV radiations $(\lambda>120 \mathrm{~nm})$ are generated from a microwave discharge hydrogen flow lamp (Opthos instruments). The fluence of the hydrogen lamp is estimated to be $c a 10^{15}$ photons $\mathrm{cm}^{-2} \mathrm{~s}^{-1}$. The concentration ratio of the different mixtures is first set before deposition using standard manometric techniques and then derived from the IR spectra by integrating vibrational bands to estimate the column density of $\mathrm{NH}_{3}$ and $\mathrm{CH}_{3} \mathrm{CHO}$ according to their band strengths provided by the literature. Moreover, the values of the band strengths depend on the nature, composition, and temperature of the ice in which they are found, and this dependence is a major source of uncertainties when evaluating the column densities of frozen molecules. For $\mathrm{NH}_{3}$, the band strengths of the wagging mode at $1070 \mathrm{~cm}^{-1}$ varies from $1.3 \times 10^{-17}$ (Kerkhof et al. 1999) to $1.7 \times 10^{-17} \mathrm{~cm}$ molecule $^{-1} \mathrm{~d}^{\prime}$ Hendecourt et al. (1986) in its pure solid form. We use the value given by Kerkhof et al. of $1.3 \times 10^{-17} \mathrm{~cm}$ molecule ${ }^{-1}$, as usually done in the literature. For $\mathrm{CH}_{3} \mathrm{CHO}$, we use the values given by Schutte et al. (1999) and Wexler (1967) of $1.3 \times 10^{-17} \mathrm{~cm} \mathrm{molecule}^{-1}$ for the CO stretch mode at $1715 \mathrm{~cm}^{-1}$ and $1.5 \times 10^{-18} \mathrm{~cm} \mathrm{molecule}^{-1}$ for the $\mathrm{CH}$ bending mode at $1350 \mathrm{~cm}^{-1}$. The thickness of the deposited solid films, assuming a density of $0.73 \mathrm{~g} \mathrm{~cm}^{-3}$ and $0.92 \mathrm{~g} \mathrm{~cm}^{-3}$ for $\mathrm{NH}_{3}$ and $\mathrm{H}_{2} \mathrm{O}$, respectively, is estimated to be around $0.1 \mu \mathrm{m}$, which is consistent with interstellar ice mantle thickness. We also use the band integration strengths to monitor the decrease in the $\mathrm{NH}_{3}$ and $\mathrm{CH}_{3} \mathrm{CHO}$ reactant IR-band intensities as they are consumed in the reaction, and to estimate how much of each product is formed. We also perform a vibrational analysis of aminoethanol to compute the harmonic vibrational frequencies for both the ${ }^{14} \mathrm{~N}$ and ${ }^{15} \mathrm{~N}$ isotopomers. Nevertheless, to simplify this analysis we do not take into account the effect of environment on the calculations. Calculations were performed using the Gaussian 98 package (Lee 1988; Frish 1998) at the $\mathrm{B} 3 \mathrm{LYP} / 6-311 \mathrm{G}++(2 \mathrm{~d}, 2 \mathrm{p})$ level, which is known to supply reliable predictions of vibrational wavenumbers.

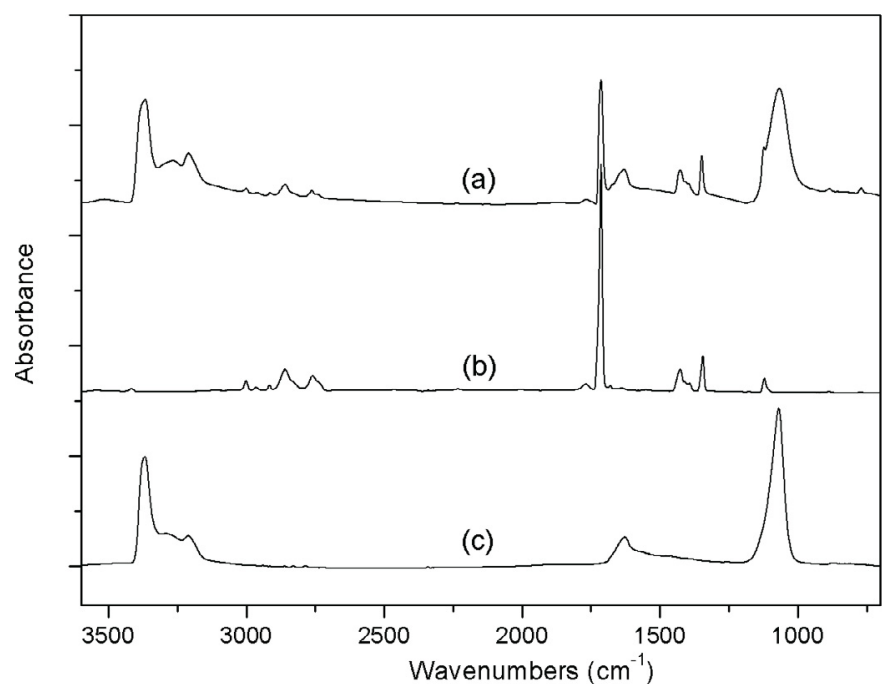

Fig. 1. Infrared spectra at $10 \mathrm{~K}$ of a) a $\mathrm{NH}_{3}: \mathrm{CH}_{3} \mathrm{CHO}$ binary mixture in a 4:1 concentration ratio; b) pure acetaldehyde; c) pure $\mathrm{NH}_{3}$.

\section{Results}

\subsection{Aminoethanol formation in a $\mathrm{NH}_{3}: \mathrm{CH}_{3} \mathrm{CHO}$ ice mixture}

Pure $\mathrm{NH}_{3}$ and acetaldehyde $\mathrm{CH}_{3} \mathrm{CHO}$ are deposited independently at $10 \mathrm{~K}$ and their respective IR spectra are displayed in Figs. $1 \mathrm{~b}$ and $\mathrm{c}$.

The assignment of each band is well known and reported in Table 1 in both pure solids and the $4: 1$ mixture. When the pure solid is heated with a temperature ramp of $4 \mathrm{~K} \mathrm{~min}^{-1}$, acetaldehyde begins to sublimate at $120 \mathrm{~K}$. On the basis of its IR signal, acetaldehyde fully disappears at $135 \mathrm{~K}$. Pure $\mathrm{NH}_{3}$ begins to sublimate at the same heating rate at $120 \mathrm{~K}$ and fully disappears at $125 \mathrm{~K}$ (Sandford \& Allamandola 1993). A binary $\mathrm{NH}_{3}: \mathrm{CH}_{3} \mathrm{CHO}$ ice mixture is then deposited at $10 \mathrm{~K}$ within a $4: 1$ concentration ratio. No reaction occurs at this temperature and the IR spectrum of this mixture is simply the superimposition of the $\mathrm{NH}_{3}$ and acetaldehyde spectra as seen in Fig. 1a. Assignments of the observed bands are listed in Table 1 . When the sample is warmed above $110 \mathrm{~K}, \mathrm{NH}_{3}$ and $\mathrm{CH}_{3} \mathrm{CHO}$ are consumed, their IR features diminish, and new features are observed as seen in Fig. 2.

After complete sublimation of the reactants, around $130 \mathrm{~K}$, the new species, the alpha-aminoethanol $\mathrm{NH}_{2} \mathrm{CH}\left(\mathrm{CH}_{3}\right) \mathrm{OH}$, formed by the reaction between acetaldehyde and ammonia remains on the gold surface. It fully sublimates at $200 \mathrm{~K}$. Its infrared spectrum at $185 \mathrm{~K}$ (Fig. 2d) provides evidence of a $\mathrm{NH}_{2}$ group at 3347 and $3285 \mathrm{~cm}^{-1}$ (Shutte et al. 1993) both $-\mathrm{CH}$ and $-\mathrm{CH}_{3}$ groups at 2978, 2934, and $2865 \mathrm{~cm}^{-1}$, and C-O/C$\mathrm{N}$ groups at 1134, 1101, and $925 \mathrm{~cm}^{-1}$ (Shutte et al. 1993). An $\mathrm{OH}$ group may be present around $3300 \mathrm{~cm}^{-1}$ but covered by broad $\mathrm{NH}_{2}$ and $\mathrm{CH}$ features. The observed bands are listed in Table 2 with their corresponding assignments. This result is comparable to the thermally promoted reaction of formaldehyde with ammonia at low temperature which leads to the formation of aminomethanol $\mathrm{NH}_{2} \mathrm{CH}_{2} \mathrm{OH}$ (Courmier et al. 2005; Bossa et al. 2009b; Woon 1999). The infrared spectrum of alphaaminoethanol is similar to that of aminomethanol, as seen in Fig. 3, especially in the NH stretching and bending regions. The upshift observed for the intense $\mathrm{C}$ - O stretching mode of alphaaminoethanol $\left(1101 \mathrm{~cm}^{-1}\right)$ with respect to the aminomethanol frequency $\left(1040 \mathrm{~cm}^{-1}\right)$ is in agreement with the difference expected between primary and secondary alcohols. The proposed mechanism for this reaction is a nucleophilic attack by the lone 
Table 1. : Infrared absorption bands and vibrational assignments of $\mathrm{NH}_{3}$ and $\mathrm{CH}_{3} \mathrm{CHO}$, in pure solids, in a $\mathrm{NH}_{3}: \mathrm{CH}_{3} \mathrm{CHO}_{\text {ice }}$ mixture and a water ice environment at $10 \mathrm{~K}$.

\begin{tabular}{ccccc}
\hline \hline \multirow{2}{*}{ Assignement } & \multicolumn{3}{c}{ Wavenumbers $\left(\mathrm{cm}^{-1}\right)$} & Band strength $\left(\mathrm{cm} \mathrm{mole}^{-1}\right)$ \\
& Pure solid & $\mathrm{NH}_{3}: \mathrm{CH}_{3} \mathrm{CHO}$ & $\mathrm{H}_{2} \mathrm{O}: \mathrm{NH}_{3}: \mathrm{CH}_{3} \mathrm{CHO}$ & \\
\hline $\mathrm{NH}_{3}$ & & & & \\
$v(\mathrm{NH})$ & 3375 & 3370 & 1636 & \\
$v(\mathrm{NH})$ & 3210 & 3210 & 1120 & $1.3 \times 10^{-17}$ \\
$\delta(\mathrm{NH})$ & 1625 & 1631 & & \\
$\omega(\mathrm{NH})$ & 1075 & 1067 & & \\
Acetaldehyde & & & & \\
$v(\mathrm{CH})$ & 3001 & 3001 & & \\
$v(\mathrm{CH})$ & 2917 & 2958 & 1721 & $1.3 \times 10^{-17}$ \\
$v(\mathrm{CH})$ & 2917 & 2915 & & \\
$v(\mathrm{CH})($ aldehyde $)$ & $2859-2764$ & $2860-2764$ & 1351 & \\
$v(\mathrm{C}=\mathrm{O})$ & 1715 & 1715 & & \\
$\delta(\mathrm{CH})$ & 1426 & 1426 & 1348 & \\
$\delta(\mathrm{CH})$ & 1345 & 1123 & & \\
$v(\mathrm{C}-\mathrm{C})$ & 1122 & & & \\
\hline
\end{tabular}

Notes. Vibration mode: stretching $(v)$, bending $(\delta)$.

Table 2. Experimental and theoretical frequencies (in $\mathrm{cm}^{-1}$ ) and integrated band strengths $\left(\mathrm{cm} \mathrm{molecule}{ }^{-1}\right.$ ) of pure $\left.{ }^{14} \mathrm{NH}_{2} \mathrm{CH}_{(\mathrm{CH}}\right) \mathrm{OH}$ and ${ }^{15} \mathrm{NH}_{2} \mathrm{CH}\left(\mathrm{CH}_{3}\right) \mathrm{OH}$.

\begin{tabular}{|c|c|c|c|c|c|c|}
\hline \multicolumn{4}{|c|}{ Theoretical B3LYP/6-311G++(2d,2p) } & \multicolumn{3}{|c|}{ Experimental } \\
\hline Mode & Position ${ }^{14} \mathrm{~N}$ & Intensities & $\Delta v\left(\left({ }^{14} \mathrm{~N}-{ }^{15} \mathrm{~N}\right)\right.$ & Position ${ }^{14} \mathrm{~N}$ & $\begin{array}{c}\mathrm{A} \\
\left(\mathrm{cm} \mathrm{mol}^{-1}\right)\end{array}$ & $\Delta v\left({ }^{14} \mathrm{~N}-{ }^{15} \mathrm{~N}\right)$ \\
\hline$v(\mathrm{OH})$ & 3813 & 20 & 0 & - & - & - \\
\hline$v(\mathrm{NH})$ & 3589 & 2 & 10 & 3347 & & 14 \\
\hline$v(\mathrm{NH})$ & 3499 & 0 & 5 & 3285 & & 5 \\
\hline$v\left(\mathrm{CH}_{3}\right)$ & 3110 & & 0 & 2978 & & 0 \\
\hline$v\left(\mathrm{CH}_{3}\right)$ & 3104 & 20 & 0 & 2978 & & 0 \\
\hline$v\left(\mathrm{CH}_{3}\right)$ & 3037 & 9 & 0 & 2934 & & 0 \\
\hline$v(\mathrm{CH})$ & 2987 & 35 & 0 & 2865 & & 0 \\
\hline$\delta(\mathrm{NH})$ & 1652 & 26 & 4 & 1616 & & 4 \\
\hline$\delta(\mathrm{CH})$ & 1502 & 3 & 0 & - & - & - \\
\hline$\delta(\mathrm{CH})$ & 1488 & 2 & 0 & - & - & - \\
\hline$\delta(\mathrm{CH})$ & 1442 & 40 & 1 & $1453(1453)$ & & 0 \\
\hline$\delta(\mathrm{CH})$ & 1413 & 14 & 1 & 1374 (1377) & $1.4 \times 10^{-18}$ & 0 \\
\hline$\delta(\mathrm{CH})+\delta(\mathrm{OH})$ & 1391 & 0 & 0 & - & - & - \\
\hline$\delta(\mathrm{OH})+\delta(\mathrm{CH})$ & 1292 & 50 & 0 & 1334 (1334) & & 0 \\
\hline$\rho(\mathrm{NH})$ & 1261 & $<1$ & 0 & 1244 (1248) & $1.9 \times 10^{-18}$ & 0 \\
\hline$v(\mathrm{C}-\mathrm{O})+v(\mathrm{C}-\mathrm{N})$ & 1158 & 45 & 2 & 1134 & & 0 \\
\hline$v(\mathrm{C}-\mathrm{N})+v(\mathrm{C}-\mathrm{C})$ & 1048 & 50 & 4 & $1101(1101)$ & $1.0 \times 10^{-17}$ & 1 \\
\hline$v(\mathrm{C}-\mathrm{N})+v(\mathrm{C}-\mathrm{C})$ & 1022 & 4 & 2 & & & \\
\hline$v(\mathrm{C}-\mathrm{O})+v(\mathrm{C}-\mathrm{N})$ & 953 & 30 & 2 & 925 (922) & $1.2 \times 10^{-17}$ & 5 \\
\hline$\omega\left(\mathrm{NH}_{2}\right)$ & 822 & 100 & 8 & - & - & - \\
\hline
\end{tabular}

Notes. Values into parenthesis are related to $\mathrm{NH}_{2} \mathrm{CH}\left(\mathrm{CH}_{3}\right) \mathrm{OH}$ in a $\mathrm{H}_{2} \mathrm{O}$ environment. Ab initio calculations are performed using the B3LYP method with the $6-311 \mathrm{G}++(2 \mathrm{~d}, 2 \mathrm{p})$ basis set. The theoretical intensities are normalized. Vibration mode: stretching $(v)$, bending $(\delta)$, rocking $(\rho)$, inversion $(\omega)$.

electronic pair of the ammonia $\mathrm{N}$ atom on the acetaldehyde $\mathrm{C}$ atom, followed by a proton transfer from $\mathrm{N}$ to $\mathrm{O}$ (Courmier et al. 2005). To confirm alpha-aminoethanol formation, we perform an isotopic substitution experiment using a ${ }^{15} \mathrm{NH}_{3}: \mathrm{CH}_{3} \mathrm{CHO}$ ice mixture in a $4: 1$ concentration ratio and compare these results with those obtained from our ab-initio calculation using the B3LYP $6-311 \mathrm{G}++(2 \mathrm{~d}, 2 \mathrm{p})$ basis set. The theoretical and experimental frequencies for ${ }^{14} \mathrm{~N}$ alpha-aminoethanol are listed in Table 2 along with theoretical and experimental frequency shifts between ${ }^{14} \mathrm{~N}$ and ${ }^{15} \mathrm{~N}$ isotopomers. Although harmonic calculations overestimate frequencies corresponding to X-H stretching modes (Table 2), we observe good agreement between the experimental and calculated frequencies of ${ }^{14} \mathrm{~N}$ alpha-aminoethanol
(Table 2) especially at frequencies lower than $1600 \mathrm{~cm}^{-1}$. We also compare experimental frequency shifts between ${ }^{14} \mathrm{~N}$ and ${ }^{15} \mathrm{~N}$ isotopomers with theoretical values. Calculated frequency shifts are small (smaller than $10 \mathrm{~cm}^{-1}$ ). The larger calculated shift of $10 \mathrm{~cm}^{-1}$ is for the $\mathrm{NH}$ stretching mode, which is in good agreement with the observed experimental shift of $14 \mathrm{~cm}^{-1}$ (Table 2). Small differences between experimental and theoretical values are observed, particularly in the region related to the $\mathrm{C}-\mathrm{O} / \mathrm{C}-\mathrm{N}$ stretching mode. From the calculations, three strong bands are expected in this region at 1158,1048 , and $953 \mathrm{~cm}^{-1}$ (Table 2). The calculated isotopic shifts are 2, 4, and $2 \mathrm{~cm}^{-1}$, respectively. Experimentally, three bands are observed in this 


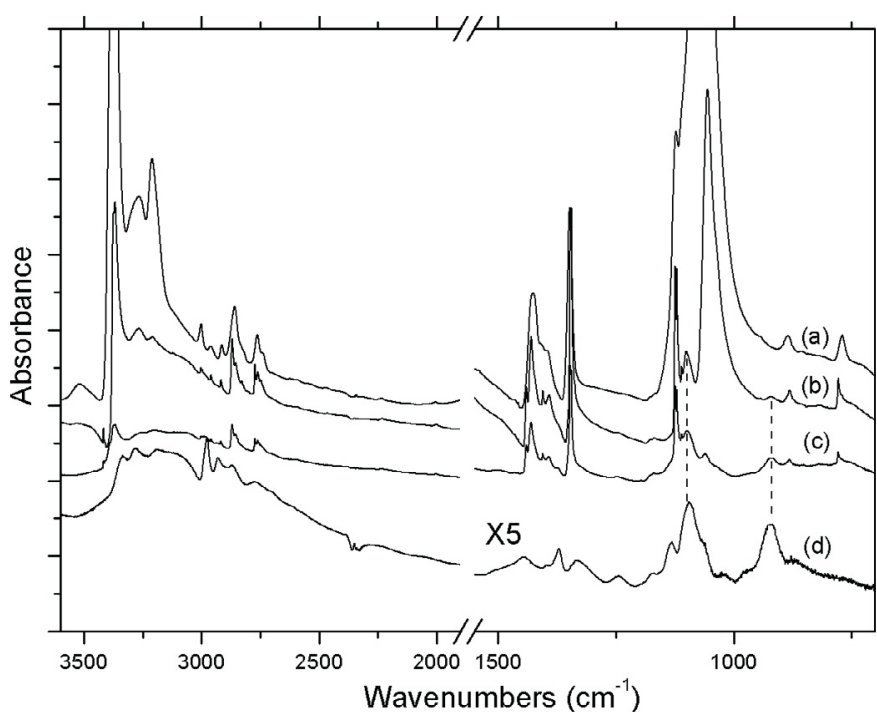

Fig. 2. Infrared spectra of the thermal evolution of a $\mathrm{NH}_{3}: \mathrm{CH}_{3} \mathrm{CHO}$ binary mixture in a $4: 1$ ratio a) at $10 \mathrm{~K}, \mathbf{b})$ at $110 \mathrm{~K}$, c) at $130 \mathrm{~K}$, and d) at $185 \mathrm{~K}$.

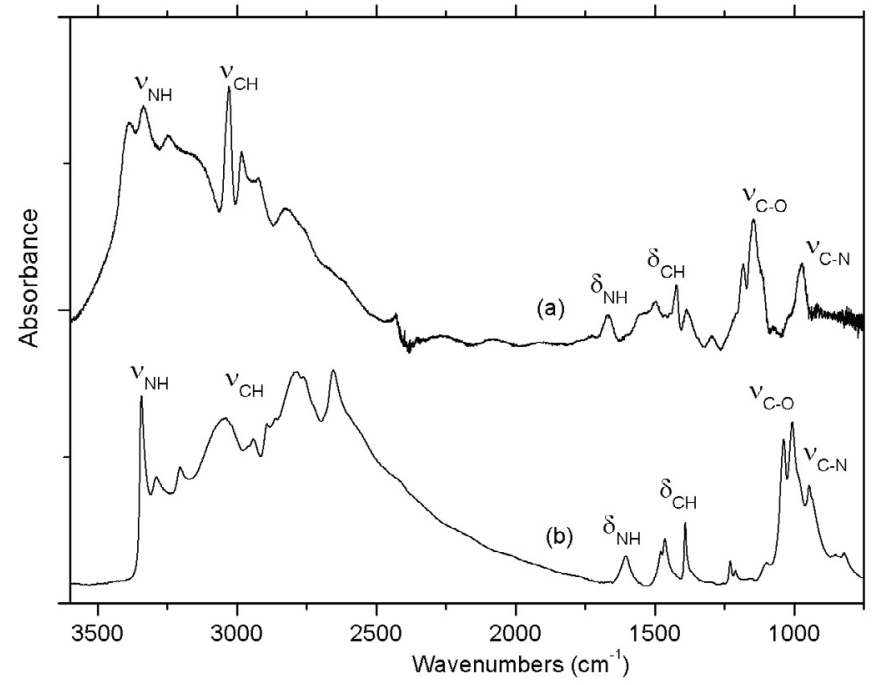

Fig. 3. Infrared spectrum at $10 \mathrm{~K}$ of a) pure alpha-aminoethanol and b) aminomethanol.

region for ${ }^{14} \mathrm{NH}_{2} \mathrm{CH}\left(\mathrm{CH}_{3}\right) \mathrm{OH}$ located at $1134,1101 \mathrm{~cm}^{-1}$ and $925 \mathrm{~cm}^{-1}$ (Fig. 2d).

The isotopic substitution induces frequency shifts of 0,1 , and $5 \mathrm{~cm}^{-1}$ respectively (Table 2) in the same order of value as the theoretical values. The alpha-aminoethanol formation is also confirmed by mass spectrometry as shown in Fig. 4. A clear peak at $m / z 46$ is observed when alpha-aminoethanol desorbs around $200 \mathrm{~K}$. It is assigned to the $\mathrm{NH}_{2} \mathrm{CHOH}^{+}$, which corresponds to the loss of a $\mathrm{CH}_{3}$ fragment from an alpha fragmentation of the alpha-aminoethanol. When the reaction is performed with ${ }^{15} \mathrm{NH}_{3}$, this peak is observed at $m / z$ 47. This result confirms that the newly formed species contains only one nitrogen atom. Unfortunately we have never observed the molecular ion at $m / z$ 61. It is probably insufficiently stable to be seen, either because alpha-aminoethanol is not stable in the gas phase or because it undergoes collisionally induced unimolecular dissociation in our experimental conditions. This is consistent with the mass spectrum of aminomethanol reported by Bossa et al. (2009a). The molecular ion of aminomethanol $\left(\mathrm{NH}_{2} \mathrm{CH}_{2} \mathrm{OH}\right)$ at

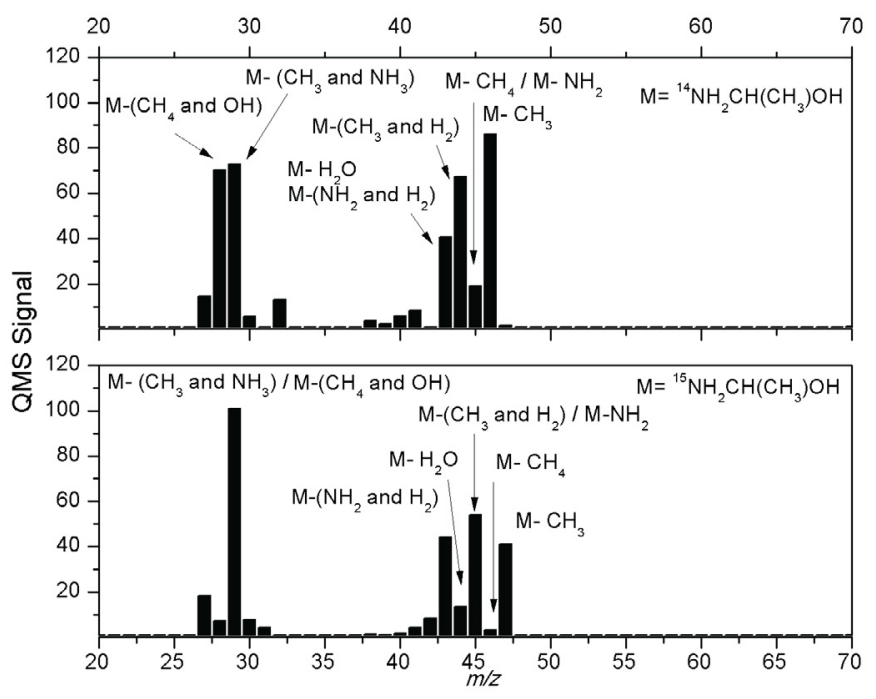

Fig. 4. The ${ }^{14} \mathrm{NH}_{2} \mathrm{CH}\left(\mathrm{CH}_{3}\right) \mathrm{OH}$ and ${ }^{15} \mathrm{NH}_{2} \mathrm{CH}\left(\mathrm{CH}_{3}\right) \mathrm{OH}$ mass spectra obtained at $200 \mathrm{~K}$ using a quadrupole mass spectrometer with a $70 \mathrm{eV}$ impact electronic source. $\mathrm{M}$ is the mass of the molecular ion of $\mathrm{NH}_{2} \mathrm{CH}\left(\mathrm{CH}_{3}\right) \mathrm{OH} \mathrm{m} / z 61$.

$\mathrm{m} / \mathrm{z}, 47$ was 15 times lower in intensity than the base peak at $\mathrm{m} / \mathrm{z}, 46$ corresponding to the loss of one hydrogen atom in the alpha position. If we were to apply the same fragmentation pattern to alpha-aminoethanol, the molecular ion would have an intensity lower than the detection threshold of our spectrometer. Moreover in molecule-bearing heteroatoms such as amines and alcohols, the most common means of fragmentation is the $\mathrm{C}-\mathrm{C}$ bond cleavage in alpha position of the heteroatom. In addition, the peak at $m / z 43$ (Fig. 4) corresponding to the loss of $\mathrm{H}_{2} \mathrm{O}$ (M18 ) is consistent with a molecule-bearing $\mathrm{OH}$ group. The peak at $\mathrm{m} / \mathrm{z} 28$ for ${ }^{14} \mathrm{NH}_{2} \mathrm{CH}\left(\mathrm{CH}_{3}\right) \mathrm{OH}$ is assigned to the loss of $\mathrm{CH}_{4}$ and $\mathrm{OH}$. The peak at $m / z 29$ for ${ }^{14} \mathrm{NH}_{2} \mathrm{CH}\left(\mathrm{CH}_{3}\right) \mathrm{OH}$ is assigned to the loss of $\mathrm{CH}_{3}$ and $\mathrm{NH}_{3}$. In the ${ }^{15} \mathrm{~N}$ isotopic experiment, they are both observed at $m / z 29$, which explains the strong intensity of this peak in the ${ }^{15} \mathrm{~N}$ isotopic experiment. The assigned mass spectra for both ${ }^{14} \mathrm{~N}$ and ${ }^{15} \mathrm{~N}$ experiments are shown in Fig. 4

\subsection{Alpha-aminoethaol formation in a water ice environment}

To form alpha-aminoethanol in a more relevant astrophysical environment, we use a water-ice-dominated $\mathrm{H}_{2} \mathrm{O}: \mathrm{NH}_{3}: \mathrm{CH}_{3} \mathrm{CHO}$ mixture. Figure 5 shows the thermal evolution of a ice mixture in a 10:5:0.5 concentration ratio deposited at $10 \mathrm{~K}$. At this temperature, the spectrum is dominated by strong water absorption bands at 3225,1628 , and $790 \mathrm{~cm}^{1}$, assigned to the $\mathrm{OH}$ stretching mode, $\mathrm{OH}$ bending mode, and libration mode, respectively. Acetaldehyde is characterized by two strong bands located at 1721 , and $1351 \mathrm{~cm}^{1}$ which can be assigned to the $\mathrm{C}=\mathrm{O}$ stretching mode and $\mathrm{CH}$ bending mode, respectively. Finally, ammonia is characterized by its strong band at $1120 \mathrm{~cm}^{1}$ assigned to the inversion mode. All the observed bands are listed in Table 1.

As previously, when the ice mixture is warmed above $100 \mathrm{~K}$, new bands at $1377,1248,1101$, and $922 \mathrm{~cm}^{1}$ corresponding to alpha-aminoethanol are observed, while the bands corresponding to ammonia and acetaldehyde decrease as can be seen in Fig. 5. The new features continue to increase in intensity until all acetaldehyde is consumed. This indicates that alphaaminoethanol is formed by a reaction involving acetaldehyde and ammonia during the warming up. At $200 \mathrm{~K}$, both unreacted 


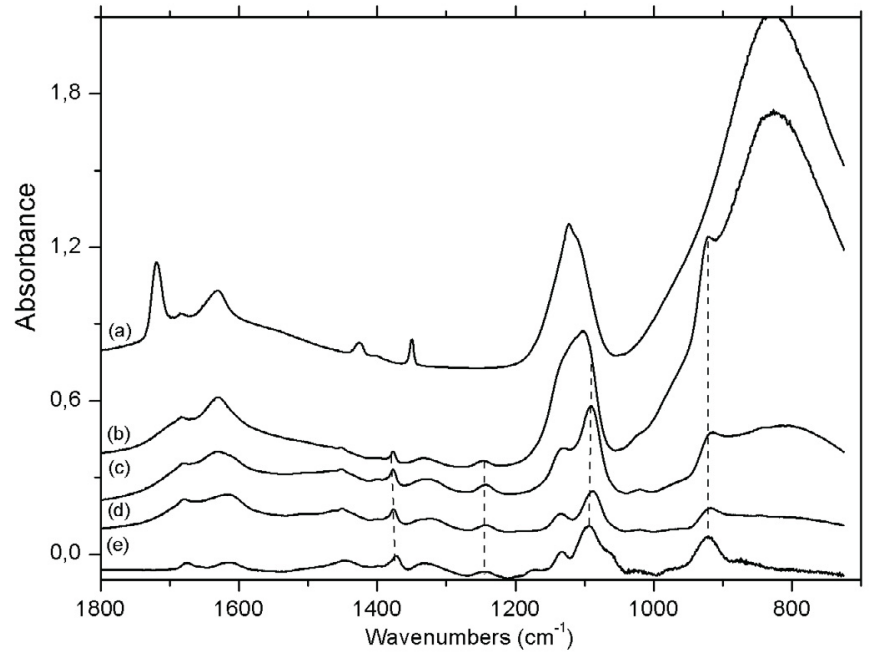

Fig. 5. FTIR spectra of a $\mathrm{H}_{2} \mathrm{O}: \mathrm{NH}_{3}: \mathrm{CH}_{3} \mathrm{CHO}$ ice in a 10:5:0.5 concentration ratio at a) $100 \mathrm{~K}$, b) $130 \mathrm{~K}$, c) $143 \mathrm{~K}$, d) $185 \mathrm{~K}$ and e) FTIR spectrum of pure alpha-aminoethanol at $185 \mathrm{~K}$ formed from a binary $\mathrm{NH}_{3}: \mathrm{CH}_{3} \mathrm{CHO}$ ice within a $4: 1$ concentration ratio.

starting material $\left(\mathrm{NH}_{3}\right.$ and $\left.\mathrm{CH}_{3} \mathrm{CHO}\right)$ and alpha-aminoethanol desorb with $\mathrm{H}_{2} \mathrm{O}$. In these conditions, it is impossible to obtain the IR spectrum of the pure product. Nevertheless, the direct comparison of the product at $185 \mathrm{~K}$ in a water environment (Fig. 5d) with the spectrum of pure alpha aminoethanol recorded at $185 \mathrm{~K}$ allow us to conclude to its formation in a water ice environment.

\subsection{The rate of alpha-aminoethanol formation}

We measure the $\mathrm{NH}_{2} \mathrm{CH}\left(\mathrm{CH}_{3}\right) \mathrm{OH}$ formation rate in a $\mathrm{H}_{2} \mathrm{O}: \mathrm{NH}_{3}: \mathrm{CH}_{3} \mathrm{CHO}$ ice mixture, where $\mathrm{NH}_{3}$ and $\mathrm{CH}_{3} \mathrm{CHO}$ are diluted into $\mathrm{H}_{2} \mathrm{O}$. The reaction rate can be written as $v=$ $k\left[\mathrm{NH}_{3}\right]^{\alpha}\left[\mathrm{CH}_{3} \mathrm{CHO}\right]^{\beta}$, where $\alpha$ and $\beta$ are the partial orders of the reaction related to $\mathrm{NH}_{3}$ and $\mathrm{CH}_{3} \mathrm{HCO}$, and $\left[\mathrm{NH}_{3}\right]$ and $\left[\mathrm{CH}_{3} \mathrm{CHO}\right]$ the molar fraction of $\mathrm{NH}_{3}$ and $\mathrm{CH}_{3} \mathrm{CHO}$, respectively. We use a $\mathrm{H}_{2} \mathrm{O}: \mathrm{NH}_{3}: \mathrm{CH}_{3} \mathrm{CHO}$ mixture in a 10:5:1 concentration ratio and measure the reaction rate as a function of temperature. The $\mathrm{H}_{2} \mathrm{O}: \mathrm{NH}_{3} \mathrm{CH}_{3} \mathrm{CHO}$ mixture is as quickly as possible brought to a fixed temperature, and the evolution with time of the species IR bands is recorded at this fixed temperature. From both the disappearance of $\mathrm{CH}_{3} \mathrm{CHO}$ and the formation of $\mathrm{NH}_{2} \mathrm{CH}\left(\mathrm{CH}_{3}\right) \mathrm{OH}$ along with time, we derive a reaction rate $k^{\prime}$, where $k^{\prime}=k\left[\mathrm{NH}_{3}\right]^{\alpha}$, considering that $\left[\mathrm{NH}_{3}\right]$ is constant since $\mathrm{NH}_{3}$ is in excess within the mixture. The single exponential decay of $\mathrm{CH}_{3} \mathrm{CHO}$ indicates a first partial order for $\mathrm{CH}_{3} \mathrm{CHO}(\beta=1)$. As for $\mathrm{NH}_{3}$, the partial order $\alpha$ is derived to be $0.2 \pm 0.01$ (Fig. 6), from reaction rate measurements at identical temperature $(T=120 \mathrm{~K})$ and different $\mathrm{NH}_{3}$ molar fractions, as seen in Table 3. Finally, the reaction is found to have a rate law $v=k\left[\mathrm{NH}_{3}\right]^{0.2}\left[\mathrm{CH}_{3} \mathrm{CHO}\right]$ and Table 3 gives the reaction rates measured for different temperatures.

This set of reaction rates as a function of temperature is fitted to an Arrhenius law $k(T)=A \exp \left(-E_{\mathrm{a}} / R T\right)$, where $E_{\mathrm{a}}$ is the activation energy and $\mathrm{A}$ is the pre-exponential factor, as shown in Fig. 7. We find that $E_{\mathrm{a}}=33 \pm 2 \mathrm{kJmol}^{-1}, A=(7 \pm 1) \times 10^{10} \mathrm{~s}^{-1}$. Introducing a temperature dependence as a power law of the preexponential factor does not improve the fit. These two parameters $E_{\mathrm{a}}$ and A can later be incorporated into kinetic databases for
Table 3. Reaction rates $k(T)$ measured for a $\mathrm{H}_{2} \mathrm{O}: \mathrm{NH}_{3}$ : $\mathrm{CH}_{3} \mathrm{CHO}$ ice mixtures at fixed temperatures.

\begin{tabular}{cccc}
\hline \hline temperature $(\mathrm{K})$ & $k^{\prime}\left(\mathrm{s}^{-1}\right)$ & {$\left[\mathrm{NH}_{3}\right]$} & $k\left(\mathrm{~s}^{-1}\right)=k^{\prime} /\left[\mathrm{NH}_{3}\right]^{0.2}$ \\
\hline 115 & $6 \times 10^{-5}$ & 0.40 & $7.2 \times 10^{-5}$ \\
120 & $2.5 \times 10^{-4}$ & 0.43 & $2.9 \times 10^{-4}$ \\
120 & $2.8 \times 10^{-4}$ & .48 & $3.2 \times 10^{-4}$ \\
120 & $2.5 \times 10^{-4}$ & .29 & $3.2 \times 10^{-4}$ \\
120 & $2.3 \times 10^{-4}$ & .19 & $3.2 \times 10^{-4}$ \\
122 & $3.7 \times 10^{-4}$ & .35 & $4.5 \times 10^{-4}$ \\
125 & $1.0 \times 10^{-3}$ & 0.41 & $21.2 \times 10^{-3}$ \\
\hline
\end{tabular}

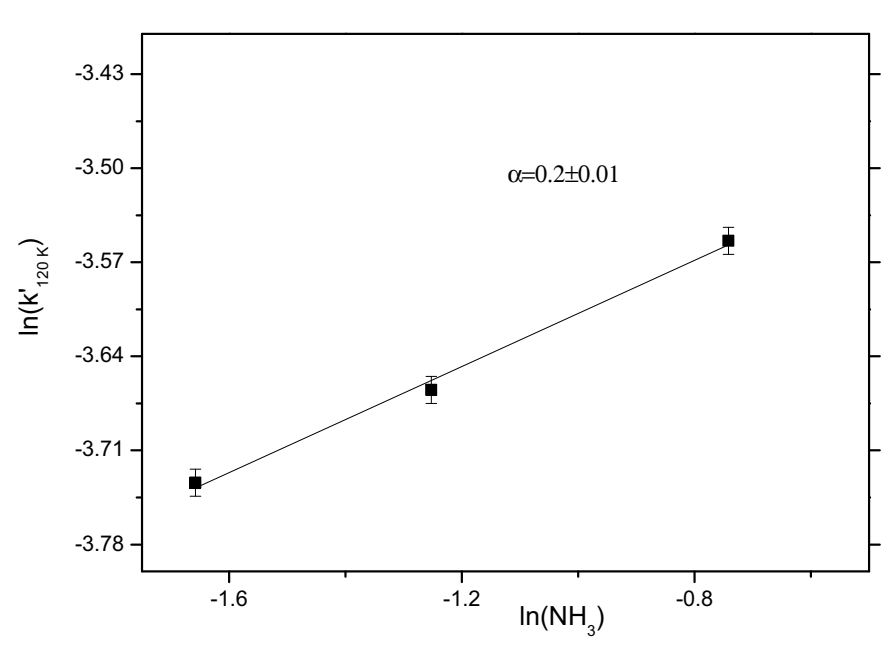

Fig. 6. The partial order $\alpha$ is derivated by measuring the $\ln \left(k^{\prime}\right)$ at $120 \mathrm{~K}$ as a function of $\ln \left(\mathrm{NH}_{3}\right)$

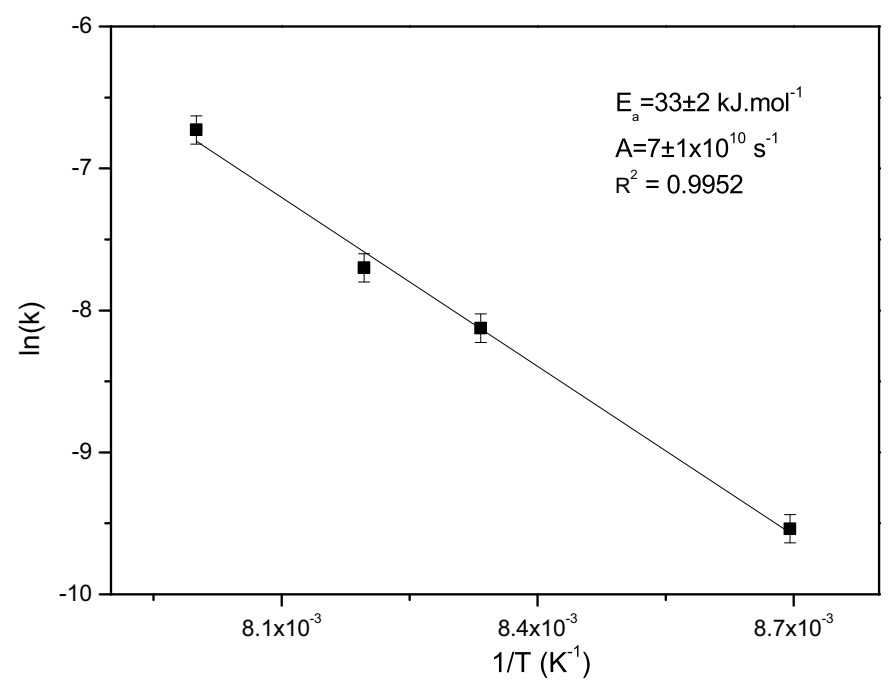

Fig. 7. The Arrhenius plot of $\ln (k)$ against $1 / T$ for the formation of alpha-aminoethanol and the best-fit straight line. The slope infers $-E_{a} / R$ and the intercept at $1 / T=0$ infers $\ln (A)$.

astrochemistry and used to extrapolate the rate of the reaction to lower temperature, assuming that Arrhenius law remains valid.

\subsection{VUV irradiation of alpha-aminoethanol}

We form $\mathrm{NH}_{2} \mathrm{CH}\left(\mathrm{CH}_{3}\right) \mathrm{OH}$ from a $\mathrm{NH}_{3}: \mathrm{CH}_{3} \mathrm{CHO}$ ice mixture in a $4: 1$ concentration ratio as described previously. We then desorb the unreacted starting material $\left(\mathrm{CH}_{3} \mathrm{CHO}\right.$ and $\left.\mathrm{NH}_{3}\right)$ at $130 \mathrm{~K}$ to obtain pure alpha-aminoethanol. We then irradiate it at 


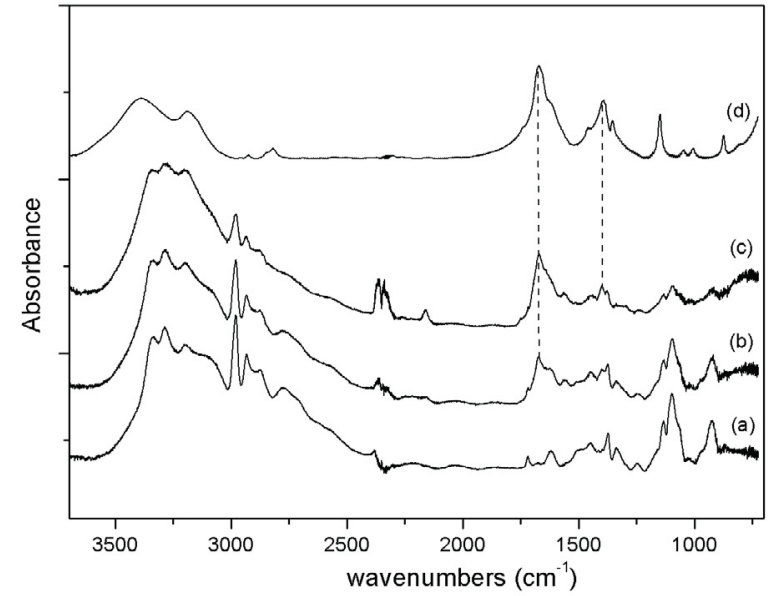

Fig. 8. FTIR spectra of a) pure alpha-aminoethanol at $135 \mathrm{~K}$ before irradiation, b) after 15 min, c) 120 min irradiation, d) acetamide in $\mathrm{KBr}$ pellet.

$10 \mathrm{~K}$ with a VUV hydrogen lamp and follow its photolysis by IR spectroscopy as seen in Fig. 8. The VUV flux is estimated to be around $10^{15}$ photons $\mathrm{cm}^{-2} \mathrm{~s}^{-1}$. After $120 \mathrm{~min}$ of VUV irradiation, $80 \%$ of alpha-aminoethanol is consumed as shown in Fig. 9 and its decay is fitted by a first order kinetic rate. The corresponding kinetic constant is found to be $4.5 \times 10^{-4} \mathrm{~s}^{-1}$. With our flux of photons, the photodissociation cross-section can be estimated to be $\sigma_{\text {photo }}=4.5 \times 10^{-19}$ photon $^{-1} \mathrm{~cm}^{2}$. Besides the IR signatures of alpha-aminoethanol (see Fig. 8), the irradiated sample displays the presence of new bands at 1674 and $1400 \mathrm{~cm}^{1}$ due to the formation of acetamide $\mathrm{CH}_{3} \mathrm{CONH}_{2}$ (Sankar et al. 1987), as shown in Fig. 8. The formation of acetamide after irradiation is also confirmed by mass spectroscopy. After the irradiation of aminoethanol at $10 \mathrm{~K}$, the sample is warmed to $250 \mathrm{~K}$. The desorption of the product at $220 \mathrm{~K}$ shows a peak at $\mathrm{m} / \mathrm{z} .59$ corresponding to the molecular ion of acetamide, $\mathrm{CH}_{3}(\mathrm{CO}) \mathrm{NH}_{2}$. This peak is not present when $\mathrm{NH}_{2} \mathrm{CH}\left(\mathrm{CH}_{3}\right) \mathrm{OH}$ is not irradiated. Its formation from alpha-aminoethanol may be the result of a dehydrogenation process as follows:

$\mathrm{NH}_{2} \mathrm{CH}\left(\mathrm{CH}_{3}\right) \mathrm{OH}+\mathrm{h} v \rightarrow \mathrm{CH}_{3} \mathrm{CONH}_{2}+\mathrm{H}_{2}$.

The same dehydrogenation mechanism has been observed in the VUV photolysis of methanol and aminomethanol that give formaldehyde and formamide, respectively (Gerakines et al. 1996; Bossa et al. 2009b). The direct production of acetamide from the photolysis of our product is additional evidence of the alpha-aminoethanol formation. The $2160 \mathrm{~cm}^{-1}$ band is easily assigned to $\mathrm{OCN}^{-}$(Hudson et al. 2000; Raunier et al. 2004) a secondary photoproduct formed by acetamide photolysis. It results from the reaction at $10 \mathrm{~K}$ between $\mathrm{HNCO}$ and $\mathrm{CH}_{3} \mathrm{NH}_{2}$, but also with the non-desorbed $\mathrm{NH}_{3}$ (Raunier et al. 2003) by means of two major channels (Duvernay et al. 2007):

$\mathrm{CH}_{3} \mathrm{CONH}_{2}+\mathrm{h} v \rightarrow \mathrm{HNCO}+\mathrm{CH}_{4}$

$\mathrm{CH}_{3} \mathrm{CONH}_{2}+\mathrm{h} v \rightarrow \mathrm{CO}+\mathrm{CH}_{3} \mathrm{NH}_{2}$.

\section{Discussion}

The $\mathrm{NH}_{3}: \mathrm{CH}_{3} \mathrm{CHO}$ ice mixture at low temperature leads to the formation of alpha-aminoethanol $\mathrm{NH}_{2} \mathrm{CH}\left(\mathrm{CH}_{3}\right) \mathrm{OH}$. This product identification is supported by (1) the agreement between the experimental and theoretical IR band shifts of

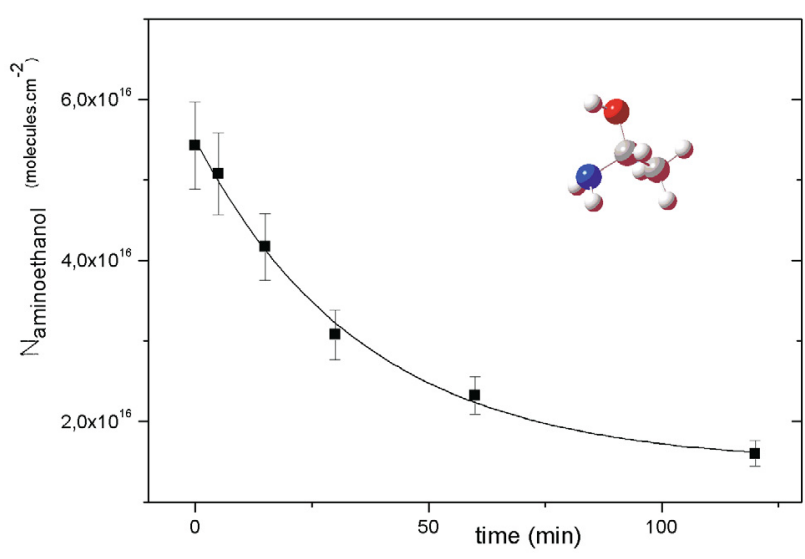

Fig. 9. VUV irradiation of aminoethanol at $135 \mathrm{~K}$. The decrease in column density is accounted for by an exponential decay.

${ }^{14} \mathrm{NH}_{2} \mathrm{CH}\left(\mathrm{CH}_{3}\right) \mathrm{OH}$ and ${ }^{15} \mathrm{NH}_{2} \mathrm{CH}\left(\mathrm{CH}_{3}\right) \mathrm{OH}$; (2) mass spectrometry results, which are consistent with alpha-aminoethanol formation; and (3) the detection of acetamide $\mathrm{CH}_{3} \mathrm{CONH}_{2}$ as VUV photolysis product. This new mechanism for acetamide production is interesting from an astrophysical point of view. Acetamide has been already detected in the interstellar medium in the gas phase and several others mechanisms have been proposed to explain its gas phase formation (Quan \& Herbst 2007; Hollis et al. 2006). Here we propose a new mechanism in the solid phase, starting with the thermal reaction between acetaldehyde and ammonia followed by VUV photolysis of the product:

$$
\mathrm{NH}_{3}+\mathrm{CH}_{3} \mathrm{CHO} \rightarrow \mathrm{NH}_{2} \mathrm{CH}\left(\mathrm{CH}_{3}\right) \mathrm{OH}+\mathrm{h} \nu \rightarrow \mathrm{CH}_{3} \mathrm{CONH}_{2}+\mathrm{H}_{2} \text {. }
$$

Thermal synthesis of aminoethanol $\mathrm{NH}_{2} \mathrm{CH}\left(\mathrm{CH}_{3}\right) \mathrm{OH}$, is the first synthesis of a chiral molecule (i.e. carbon with four different substituents) in interstellar ice analogs without any nonthermal energetic processes (i.e. photon irradiation or particle bombardment). However, we note that in our conditions the alpha-aminoethanol will be produced as a racemate.

Chiral molecules have an essential role in prebiotic chemistry and many biologically active molecules, including aminoacids (the building blocks of proteins) and sugars, have the same chirality. Thus, the possibility of alpha-aminoethanol formation in interstellar ices is interesting to consider as it may be an intermediate in the formation of more complex chiral molecules such as alanine, one of the most common aminoacids. Alanine $\mathrm{NH}_{2} \mathrm{CH}\left(\mathrm{CH}_{3}\right) \mathrm{COOH}$ would be the product of the Strecker synthesis that can be summarized by two steps: (i) a thermal reaction between $\mathrm{CH}_{3} \mathrm{CHO}$ and $\mathrm{NH}_{3}$ to form alphaaminoethanol; and (ii) an acid hydrolysis of alpha-aminoethanol in the presence of $\mathrm{HCN}$ to produce successively aminoacetonitrile and finally alanine. Here alanine would be again formed as a racemate. The first question is in which astrophysical environments can we envisage the presence of aminoethanol. $\mathrm{NH}_{3}$ has been detected in interstellar ices through different lines of sight (mainly high-mass young stellar objects YSOs, such as W33A) with a $10 \%$ maximum abundance relative to $\mathrm{H}_{2} \mathrm{O}$. It has also been detected in the gas phase of the interstellar medium or in cometary ices. Acetaldehyde has been detected in the gas phase of the interstellar medium and in comets but its detection still requires confirmation in interstellar ices. The presence of this molecule in ices should not exceed 1\% (Shutte et al. 1999; Gibb et al. 2004; Dartois 2005). 


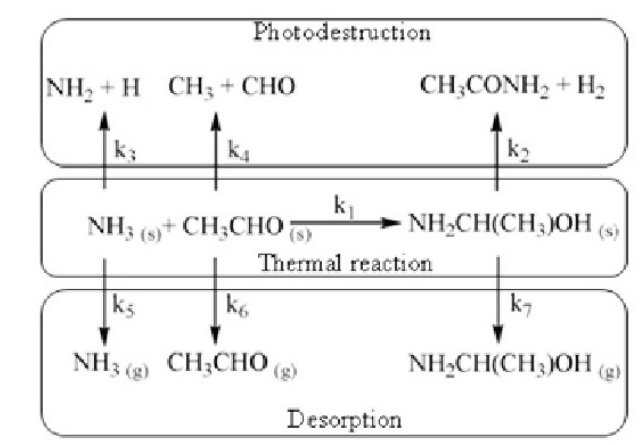

$$
\begin{aligned}
& \frac{d N_{N H_{3}}}{d t}=-k_{1} N_{N H_{3}} \cdot N_{C H_{3} C H O}-k_{3} N_{N H_{3}}-k_{5} N_{N H_{3}} \\
& \frac{d N_{\mathrm{CH}_{3} \mathrm{CHO}}}{d t}=-k_{1} N_{\mathrm{NH}_{\mathrm{g}}} \cdot \mathrm{N}_{\mathrm{CH}_{8} \mathrm{CHO}}-k_{4} N_{\mathrm{CH}_{\mathrm{g}} \mathrm{CHO}}-k_{6} \mathrm{~N}_{\mathrm{CH}_{3} \mathrm{CHO}} \\
& \frac{d N_{\mathrm{NH}_{2} \mathrm{CH}\left(\mathrm{CH}_{\mathrm{s}}\right) \mathrm{OH}}}{d t}=k_{1} N_{\mathrm{NH}_{\mathrm{s}}} \cdot \mathrm{N}_{\mathrm{CH}_{\mathrm{s}} \mathrm{CHO}}-k_{2} \mathrm{~N}_{\mathrm{NH}_{2} \mathrm{CH}\left(\mathrm{CH}_{\mathrm{s}}\right) \mathrm{OH}}-k_{7} \mathrm{~N}_{\mathrm{NH}} \mathrm{CH}\left(\mathrm{CH}_{\mathrm{s}}\right) \mathrm{OH}
\end{aligned}
$$

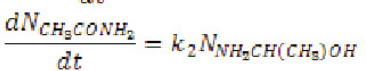

Fig. 10. Simplified chemical reaction network and the ordinary differential equation system used to model the alpha-aminoethanol production.

Alpha-aminoethanol may be formed thermally in objects that are mildly warm, such as YSOs, or comets and we roughly estimated its production in these environments using a simple model. To determine the alpha-aminoethanol abundance as a function of time in different environments such as cold molecular clouds, comets, or YSOs we needed to numerically solve a system of first-order differential equations to model the formation and destruction of each species, the abundance of water being kept constant. These equations can be sorted into three different classes: thermal formation, thermal desorption, and photodestruction. The chemical reaction network and the ordinary differential equation system are shown in Fig. 10. For two-body thermal reactions $\left(k_{1}\right)$ and thermal desorptions $\left(k_{5}, k_{6}, k_{7}\right)$, the rate coefficients are given under the form $k=A \cdot \exp (-E / R T)$, where $T$ is the ice temperature, $A$ the pre-exponential factor, and $E$ the energy (activation or desorption). Photodissociation rates $\left(k_{2}, k_{3}, k_{4}\right)$ are written as $k=\sigma . f$, where $\sigma$ represents the photodissociation cross-section and $f$ the interstellar ultraviolet radiation field (Woodall et al. 2007). We used kinetic data as well as the photodissociation cross-section obtained in this present work to estimate the kinetics rates of the formation $\left(k_{1}\right)$ and photodissociation reactions $\left(k_{2}\right)$ of alpha-aminoethanol. We also took into account the photodestruction rates of $\mathrm{NH}_{3}$ $\left(k_{3}\right)$ and $\mathrm{CH}_{3} \mathrm{CHO}\left(k_{4}\right)$ reactants using the kinetic rates of the UMIST database (Woodall et al. 2007). We included the desorption of the $\mathrm{NH}_{3}\left(k_{5}\right)$ and $\mathrm{CH}_{3} \mathrm{CHO}\left(k_{6}\right)$ reactants, and the $\mathrm{NH}_{2} \mathrm{CH}\left(\mathrm{CH}_{3}\right) \mathrm{OH}$ product $\left(k_{7}\right)$. For the $k_{5}$ and $k_{6}$ we used the value given by Sandford \& Allamandola (1993). The desorption rate of aminethanol $\left(k_{7}\right)$ was estimated from the measured desorption energy of aminomethanol (Bossa et al. 2009a), since they both desorb at the same temperature. The initial abundance for $\mathrm{NH}_{3}$ and $\mathrm{CH}_{3} \mathrm{CHO}$ are $10 \%$ and $1 \%$ with respect to $\mathrm{H}_{2} \mathrm{O}$ respectively. All thermal formation, photodestruction, and desorption kinetic rates are given in Table 4.

Figure 11 shows the solid alpha-aminoethanol abundance relative to $\mathrm{H}_{2} \mathrm{O}$ evolution as a function of time, temperature, and UV flux. With a flux of $10^{4}$ photon $\mathrm{cm}^{-2} \mathrm{~s}^{-1}$, typical of attenuated field within a dense molecular cloud, the amount of alpha-aminoethanol is maximal (around 1\% with respect to $\mathrm{H}_{2} \mathrm{O}$ ) within a month and $10^{5}$ years in the range 90-110 K, and within 1000 years at $130 \mathrm{~K}$. The maximum amount of

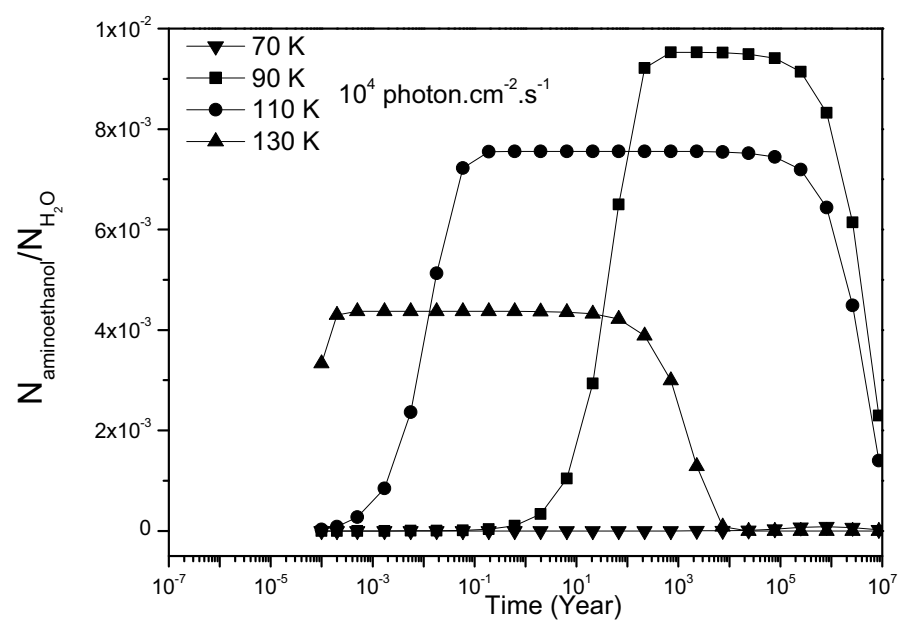

Fig. 11. Alpha-aminoethanol abundance in ice as a function of time, temperature, and flux of VUV photon $\left(10^{4}\right.$ photon $\left.\mathrm{cm}^{-2} \mathrm{~s}^{-1}\right)$ as obtained by solving our simple model.

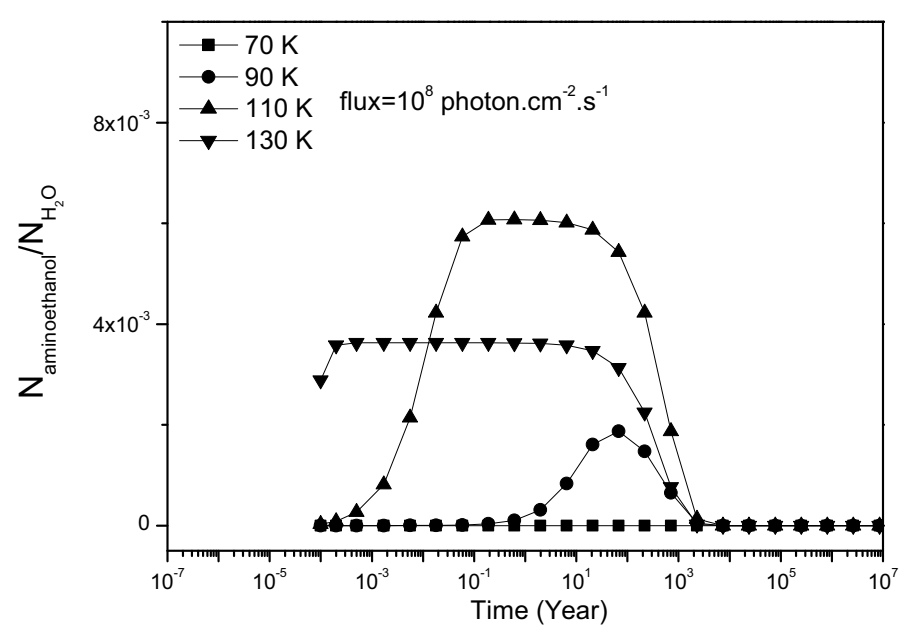

Fig. 12. Alpha-aminoethanol abundance in ice as a function of time, temperature, and flux of VUV photon $\left(10^{8}\right.$ photon $\left.\mathrm{cm}^{-2} \mathrm{~s}^{-1}\right)$ as obtained by solving our simple model.

alpha-aminoethano occurs at $90 \mathrm{~K}$. With a flux of VUV photons around $10^{8}$ photon $\mathrm{cm}^{-2} \mathrm{~s}^{-1}$ (Fig. 12), typical of the average interstellar radiation field in the diffuse medium, alphaaminoethanol cannot be observed after 1000 years regardless of the temperature because of the photodissociation process. Therefore, the best conditions to observe solid-state alphaaminoethanol would be a warm $(T>80 \mathrm{~K})$ and blended environment $\left(f<10^{5}\right.$ photon $\left.\mathrm{cm}^{-2} \mathrm{~s}^{-1}\right)$ as in the warm layer of a protoplanetary disk or in a hot corino. A cometary nucleus may be exposed to a wide range of temperatures anda VUV radiation field, depending on its distance to the star, so a cometary nucleus could also be a suitable environment for alpha-aminoethanol formation. $\mathrm{NH}_{3}$ and $\mathrm{CH}_{3} \mathrm{CHO}$ have already been detected in cometary coma, so the thermal reaction between them may lead to the formation of alpha-aminoethanol in this environment. However approximate this reduced model may be, it provides a rough idea of what the favorable conditions are to produce alphaaminoethanol. It can be produced on a reasonable time-scale in relevant interstellar conditions, and thus may be a plausible candidate for further investigation of cometary material. Moreover, if it is stable in the gas phase, alpha-aminoethanol may be observed from its rotational transitions in hot core regions when the solid material is desorbed in the gas phase. 
Table 4. Parameters for thermal formation, photo-destruction, and desorption rates.

\begin{tabular}{cccccc}
\hline \hline & Species & & $A\left(\mathrm{~s}^{-1}\right)$ & $E\left(\mathrm{~kJ} \mathrm{~mol}^{-1}\right)$ & Ref. \\
\hline Thermal formation rate $^{a}$ & $\mathrm{NH}_{2} \mathrm{CH}\left(\mathrm{CH}_{3}\right) \mathrm{OH}$ & $k_{1}$ & $6.7 \times 10^{10}$ & 33 & this work \\
\hline \multirow{2}{*}{ Desorption rate $^{a}$} & $\mathrm{NH}_{3}$ & $k_{5}$ & $3 \times 10^{12}$ & 25.5 & Sandford \& Allamandola (1993) \\
& $\mathrm{CH}_{3} \mathrm{CHO}$ & $k_{6}$ & $3 \times 10^{12}$ & 25.5 & Sandford \& Allamandola (1993) \\
& $\mathrm{NH}_{2} \mathrm{CH}\left(\mathrm{CH}_{3}\right) \mathrm{OH}$ & $k_{7}$ & $3 \times 10^{12}$ & 58 & Bossa et al. (2009a) \\
\hline & & \multicolumn{3}{c}{$\sigma\left(\right.$ photon $\left.^{-1} \mathrm{~cm}^{2}\right)$} & this work \\
Photo-destruction rate $^{b}$ & $\mathrm{NH}_{2} \mathrm{CH}\left(\mathrm{CH}_{3}\right) \mathrm{OH}$ & $k_{2}$ & $4.5 \times 10^{-19}$ & Woodall et al. (2007) \\
& $\mathrm{NH}_{3}$ & $k_{3}$ & $5 \times 10^{-18}$ & Woodall et al. (2007) \\
\hline
\end{tabular}

Notes. ${ }^{(a)}$ The rate coefficients are given in the form $k=A \cdot \exp (-E / R T)$, where $T$ is the ice temperature, $A$ the pre-exponential factor, and $E$ the energy (activation or desorption). ${ }^{(b)}$ Photodissociation rates are written as $k=\sigma$.f, where $\sigma$ represents the photodissociation cross-section and $f$ the interstellar ultraviolet radiation field.

\section{Conclusion}

In this work, we have investigated the thermal reaction of solid $\mathrm{NH}_{3}$ and acetaldehyde $\mathrm{CH}_{3} \mathrm{CHO}$ at low temperature. This reaction leads to the formation of a chiral molecule, the alpha aminoethanol $\mathrm{NH}_{2} \mathrm{CH}\left(\mathrm{CH}_{3}\right) \mathrm{OH}$. For the first time, we report its infrared and mass spectra. We have also investigated its photochemical behavior under VUV irradiation. The main photoproduct is acetamide $\left(\mathrm{CH}_{3} \mathrm{CONH}_{2}\right)$. The data provided in this work demonstrate that the alpha-aminoethano is formed at low temperature on a one-hour time-scale in the laboratory, suggesting that its formation in warm interstellar environments, such as protostellar envelopes or in cometary environments, is likely. The alpha-aminoethanol should be analyzed in more detail by future studies of cometary material or the gas phase by radioastronomy. The mass spectrum of the alpha-aminoethanol obtained in this study is of interest for the forthcoming analysis of the Rosetta mission: the observation of a peak at $\mathrm{m} / \mathrm{z}, 46$ from the COSAC gas analyzer maybe the signature of an aminoalcohol.

Acknowledgements. This work has been founded by the French national program Physique Chimie du Milieu Interstellaire (P.C.M.I) and the Centre National des Etudes Spatiales (C.N.E.S).

\section{References}

Bossa, J., Theule, P., Duvernay, F., Borget, F., \& Chiavassa, T. 2008, A\&A, 492, 719

Bossa, J., Duvernay, F., Theule, P., et al. 2009a, A\&A, 506, 601
Bossa, J., Theule, P., Duvernay, F., \& Chiavassa, T. 2009b, ApJ, 707, 1524

Charnley, S., Ehrenfreund, P., \& Kuan, Y. 2001, Spectrochimica acta part AMolecular and Biomolecular Spectroscopy, 57, 685

Courmier, D., Gardebien, F., Minot, C., St-Amant, A. 2005, Chem. Phys Lett., 405, 357

Cronin, J. R., Pizzarello, S. 1997, Science, 275, 951

Cronin, J. R. Pizzarello, S. 1999, Adv. Space Res., 23, 293

Dartois, E. 2005, Space Sci. Rev. 119, 293

D'Hendecourt, L. B., Allamandola, L. J. 1986, A\&ASS, 64, 453

Duvernay, F., Chatron-Michaud, P., Borget, F., Birney, D. M., \& Chiavassa, T. 2007, Phys. Chem. Chem. Phys., 9, 1099

Frisch, M., et al. 1998, GAUSSIAN 98 (Revision A.7), Gaussian Inc Pittsburgh, PA

Gerakines, P. A., Schutte, W. A., \& Ehrenfreund, P. 1996, A\&A, 312, 289

Gibb, E., Whittet, D., Boogert, A., \& Tielens, A. 2004, A\&ASS, 151, 35

Hollis, J. M., Lovas, F. J., Remijan, A. J., et al. 2006, ApJ, 643, L25

Hudson, R. L., \& Moore, M. H. 2000, A\&A, 357, 787

Kerkhof, O., Schutte, W., \& Ehrenfreund, P. 1999, A\&A, 346, 990

Lee, C. 1988, Phys. Rev. B: Condens. Matter 37, 785

Quan, D., Herbst, E. 2007, A\&A, 474, 521

Raunier, S. 2003, J. Chem. Phys. Lett., 2003, 368, 594

Raunier, S., Chiavassa, T., Marinelli, F., \& Aycard, J.-P. 2004, J. Chem. Phys., 302, 259

Sandford, S. A., \& Allamandola, L. J. 1993, ApJ, 417, 815

Sankar Bala, S., Panja, P. K., \& Ghosh, P. N. 1987, J. Mol. Structure, 157, 339

Schutte, W. A., Allamandola, L. J., \& Sandford, S. A. 1993, Icarus, 104, 118

Schutte, W., Boogert, A., Tielens, A., et al. 1999, A\&A, 343, 966

van Dishoeck, E., \& Blake, G. 1998, Ann. Rev. A\&A, 36, 317

Wexler, A. 1967, Appl. Spectros. Revs., 1, 29

Whittet, D., Schutte, W., Tielens, A., et al. 1996, A\&A, 315, L357

Woon, D. E. 1999, Icarus, 142, 550

OSU database

Woodall, J., Agundez, M., Markwick-Kemper, A., \& Millar, T. 2007, A\&A, 466, 1197 